\title{
Averaging for non-homogeneous switched DAEs
}

\author{
Elisa Mostacciuolo
}

Stephan Trenn $\diamond$

Francesco Vasca ${ }^{\natural}$

\begin{abstract}
Averaging is widely used for approximating the dynamics of switched systems. The validity of an averaged model typically depends on the switching frequency and on some technicalities regarding the switched system structure. For homogeneous linear switched differential algebraic equations it is known that an averaged model can be obtained. In this paper an averaging result for non-homogeneous switched systems is presented. A switched electrical circuit illustrates the practical interest of the result.
\end{abstract}

\section{INTRODUCTION}

In this paper the averaging problem for non-homogeneous systems represented by means of differential algebraic equations (DAEs) is considered. Switched DAEs systems are given by

$$
\begin{aligned}
E_{i} \dot{x}(t) & =A_{i} x(t)+B_{i} u(t) \text { on } \mathrm{t} \in\left[s_{k, i}, s_{k, i+1}\right), \\
x\left(0^{-}\right) & =x_{0} \in \mathbb{R}^{n},
\end{aligned}
$$

with $E_{i}, A_{i} \in \mathbb{R}^{n \times n}, B_{i} \in \mathbb{R}^{n \times m}$ and $s_{k, i}, s_{k, i+1}$ the switching time instants where $i=1, \ldots, q \in \mathbb{N}$, indicates the active mode. Physical systems whose state variables satisfy certain algebraic constraints alongside some differential equations, can be naturally modeled with DAEs, [1][4]. The switched DAEs arise naturally when this kind of systems change their model during the time, which occurs in many practical engineering systems [5], [6]. The analysis of switched DAEs is complicated by the interactions between the continuous dynamics of modes and the switching signal which determines the active mode. A possible way to circumvent some of these difficulties is the averaging approach; if the frequency of the periodic switching signal is high compared to the dynamics of the continuous state variables it is possible to give an explicit formulation of a (simpler) averaged model which approximates in some sense the slow dynamics of the system [7], [8]. The averaging theory for switched systems has a big interest in the control theory due to the wide field of application such as power electronic systems, multi-agent systems [9], synchronization of oscillators [10], [11], pneumatic systems [12], switched controllers [13], [14], congestion control mechanisms [15], robotic manipulators [16], nonlinear circuits [17], dynamical networks [18], biological systems [19]. Different aspects have been investigated in the literature dealing with the averaging for switched systems: harmonic analysis with periodic switchings [20], exponential splitting for modes repre-

( ) Department of Engineering, University of Sannio, 82100 Benevento, Italy, email: elisa.mostacciuolo@unisannio.it, vasca@unisannio.it

$(\diamond)$ Department of Mathematics, University of Kaiserslautern, 67663 Kaiserslautern, Germany, email: trenn@mathematik. uni-kl.de sented by ordinary differential equations (ODEs) [21], dithering [22], hybrid behaviors [23]; for an overview see [24].

The averaging for switched DAEs is a nontrivial extension of the switched ODEs case due to the presence of jumps in the solution. An averaged model for homogeneous switched DAE systems with two modes is proposed in [25], this result is extended to the case of multi-mode switched DAEs in [26] while in [27] an averaging result with relaxed assumptions is presented. Nevertheless this result cannot be directly applied to the case of non homogeneous systems in which the input function is not necessary of order of the switching period. The averaging result for non-homogeneous switched DAEs proposed in this paper is based on relaxed assumptions and is valid also for multiple modes.

The paper is organized as follows. Section II presents a brief reminder on the theory of switched DAEs. In Sec. III the averaging result is proved. In Sec. IV numerical results for a switched capacitor electrical circuit motivate the practical interest of the theoretical result. The conclusions are presented in Sec. V.

\section{Preliminaries}

Throughout the paper the following notation is used. $x\left(t^{-}\right)$ denote the left-sided limit of the $x$ at $t \in \mathbb{R}$, i.e. $x\left(t^{-}\right):=$ $\lim _{\epsilon \succ 0} x(t-\epsilon)$, while the right-sided limit $x\left(t^{+}\right)$is defined analogously; $p \in \mathbb{R}$ is the switching period, $t_{k}=k p$ is the time instant $k$-th multiple of $p$ and $s_{k, i} \in \mathbb{R}$ is the switching time instant when the mode $i$ becomes active between $t_{k}$ and $t_{k+1}$, with $k$ being a nonnegative integer.

\section{A. Property of regularity pair matrices}

A matrix pair $(E, A) \in \mathbb{R}^{n \times n} \times \mathbb{R}^{n \times n}$ is said regular, if the polynomial $\operatorname{det}(s E-A)$ is not the zero polynomial.

Proposition 1 (Quasi-Weierstrass [28], [29]): A matrix pair $(E, A) \in \mathbb{R}^{n \times n} \times \mathbb{R}^{n \times n}$ is regular if and only if there exist invertible transformation matrices $S, T \in \mathbb{R}^{n \times n}$ which put $(E, A)$ into quasi Weierstrass form

$$
(S E T, S A T)=\left(\left[\begin{array}{cc}
I & 0 \\
0 & N
\end{array}\right],\left[\begin{array}{cc}
J & 0 \\
0 & I
\end{array}\right]\right)
$$

where $N \in \mathbb{R}^{n_{2} \times n_{2}}$, with $0 \leq n_{2} \leq n$ is a nilpotent matrix, $J \in \mathbb{R}^{n_{1} \times n_{1}}$ with $n_{1}:=n-n_{2}$ is some matrix and $I$ is the identity matrix of the appropriate size.

In [29], [30] is shown that the transformation matrices $S$ and $T$ can be obtained via the so called Wong sequences, [31].

Based on the quasi-Weierstrass form one can define the following flow matrix and projectors. 
Definition 1 (Flow matrix, [32]): Consider a regular matrix pair $(E, A)$ and its quasi Weierstrass form. The flow matrix $A^{\text {diff }}$ of $(E, A)$ is given by

$$
A^{\text {diff }}=T\left[\begin{array}{ll}
J & 0 \\
0 & 0
\end{array}\right] T^{-1} .
$$

Definition 2 (Consistency, differential and impulsive projectors): Consider a regular matrix pair $(E, A)$ and its quasi Weierstrass form. The consistency projector $\Pi$, the differential projector $\Pi^{\text {diff }}$ and the impulsive projector $\Pi^{\text {imp }}$ of the matrix pair $(E, A)$ are defined respectively as follows

$$
\begin{aligned}
\Pi & :=T\left[\begin{array}{ll}
I & 0 \\
0 & 0
\end{array}\right] T^{-1}, I \in \mathbb{R}^{n_{1} \times n_{1}} \\
\Pi^{\text {diff }} & :=T\left[\begin{array}{ll}
I & 0 \\
0 & 0
\end{array}\right] S, I \in \mathbb{R}^{n_{1} \times n_{1}} \\
\Pi^{\text {imp }} & :=T\left[\begin{array}{ll}
0 & 0 \\
0 & I
\end{array}\right] S, I \in \mathbb{R}^{n_{2} \times n_{2}},
\end{aligned}
$$

From (2) and (3) the following properties can be simply verified:

$$
A^{\text {diff }}=\Pi^{\text {diff }} A, \quad E^{\text {imp }}=\Pi^{\text {imp }} E .
$$

Note that only the consistency projector is a projector in the usual sense (i.e., it is an idempotent matrix $\Pi^{j}=\Pi, j \in \mathbb{N}$ ).

Furthermore, it can be shown [33] that the projectors don't depend on the choice of the matrices $T$ and $S$ which bring to the quasi Weierstrass form of the matrix pair $(E, A)$.

\section{B. Solution theory for switched DAEs}

Consider the non-homogeneous switched DAE system (1) with $q$ modes and the switching time instants for each $k \in \mathbb{N}$ defined as follows

$$
\begin{array}{rlrl}
s_{k, 1} & :=k p, & s_{k, i} & :=k p+\sum_{j=1}^{i-1} d_{j} p \\
t_{k}:=s_{k, 1}, & t_{k+1}:=s_{k+1,1}=(k+1) p
\end{array}
$$

with $i=2, \ldots, \mathrm{q}, p>0$ the switching period and $d_{i} \in(0,1)$ the duty cycle of $i$-th mode, see Fig. 1 .

Throughout the work it is assumed that $\left(E_{i}, A_{i}\right)$ are regular matrix pairs and that the switched DAEs is impulse free. Note that impulse-freeness does not exclude jumps in the solution; for more details on impulses and distributional solutions see e.g. [5]. Furthermore some preliminary results concerning the convergence of the Dirac impulses are proved in [34]. The (impulse-free) solution of (1) is obtained by "concatenating" the solution of each mode, that can be written as follows

$$
\begin{aligned}
x(t)=e^{A_{i}^{\mathrm{diff}} t} \Pi_{i} x\left(s_{k, i}^{-}\right) & +\int_{s_{k, i}}^{t} e^{A_{i}^{\mathrm{diff}}(t-s)} \Pi_{i}^{\mathrm{diff}} B_{i} u(s) \mathrm{d} s \\
& -\sum_{i=0}^{n-1}\left(E_{i}^{\mathrm{imp}}\right)^{i} \Pi_{i}^{\mathrm{imp}} B_{i} u(t)^{(i)}
\end{aligned}
$$

with $t \in\left(s_{k, i}, s_{k, i+1}\right)$ and the initial condition of the $i$-th mode is given by

$$
x\left(s_{k, i}^{+}\right)=\Pi_{i} x\left(s_{k, i}^{-}\right)-\sum_{i=0}^{n-1}\left(E_{i}^{\mathrm{imp}}\right)^{i} \Pi_{i}^{\mathrm{imp}} B_{i} u^{(i)}\left(s_{k, i}^{+}\right)
$$

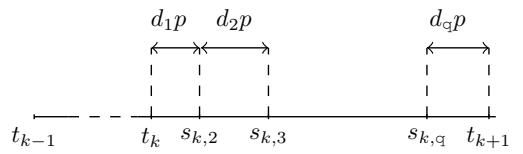

Fig. 1. Graphical representation of the time interval $\left[t_{k-1}, t_{k+1}\right]$, with $t_{k}=k p, t_{k+1}=k p+p$ and $s_{k, i}:=k p+\sum_{j=1}^{i-1} d_{j} p i=2, \ldots, q$.

where $x\left(s_{k, i}^{-}\right)$is the solution of mode $i-1$ evaluated at the time instant time $s_{k, i}^{-}$. The consistency projector $\Pi_{i}$ plays a role in the presence of possible inconsistent initial conditions at the switching time instants $s_{k, i}$, i.e., when the $i$-th mode is activated.

\section{Averaging for homogeneous switched DAEs}

Definition 3 (Big $O$ notation): Consider any functions $f:(0, \infty) \rightarrow \mathcal{V}$ and $g:(0, \infty) \rightarrow(0, \infty)$, where $\mathcal{V}$ is some normed vector space with norm $\|\cdot\|$. We say that $f(p)$ is an $\mathrm{O}(g(p))$ function $(f(p)=\mathrm{O}(g(p))$ for short), if there exist constants $\alpha$ and $\bar{p}>0$ such that

$$
\|f(p)\| \leq \alpha g(p), \quad \forall p \in(0, \bar{p}] .
$$

Given a compact set $\mathfrak{J} \in(0, \infty)$ we say that

$$
f(t)-g(t)=\mathrm{O}(p), \quad \forall t \in \mathfrak{J}
$$

if the difference of two functions is $\mathrm{O}(p)$ uniformly in $t$, i.e. the constant $\alpha$ is independent of $t$.

The following averaging result for homogeneous switched DAEs is already known.

Proposition 2 (Averaging for homogeneous switched DAEs [25]): Consider an homogeneous-switched DAE system with flow matrices $A_{i}^{\text {diff }}$ and consistency projectors $\Pi_{i}$. Assume that the consistency projectors commute, i.e.,

$$
\Pi_{i} \Pi_{j}=\Pi_{j} \Pi_{i}, \quad \forall i \neq j \in\{1, \ldots, \mathrm{q}\} .
$$

Then the corresponding averaged system is given by

$$
\dot{x}_{\mathrm{av}}=A_{\mathrm{av}} x_{\mathrm{av}}, \quad x_{\mathrm{av}}(0)=\Pi_{\cap} x_{0}
$$

where

$$
\begin{aligned}
A_{\mathrm{av}} & :=\Pi_{\cap}\left(A_{1}^{\text {diff }} d_{1}+A_{2}^{\text {diff }} d_{2}+\ldots+A_{\mathrm{q}}^{\text {diff }} d_{\mathrm{q}}\right) \Pi_{\cap}, \\
\Pi_{\cap} & :=\Pi_{\mathrm{q}} \Pi_{\mathrm{q}-1} \cdots \Pi_{2} \Pi_{1},
\end{aligned}
$$

and $x_{0} \in \mathbb{R}^{n}$. Consider an arbitrary constant $\Delta>p$, then the following holds

$$
x_{\sigma, p}(t)-x_{\mathrm{av}}(t)=\mathrm{O}(p) \forall t \in[p, \Delta]
$$

where $x_{\sigma, p}$ denotes the (impulse-free) solution of the homogeneous switched system and $x_{\mathrm{av}}$ is the (continuously differentiable) solution of (8).

Note that if the projectors commute then the amplitude of the state jumps converge to zero when the switching period tends to zero. Indeed in the limit the solution of the switched DAE coincides with a solution of an ODE (which does not exhibit jumps). 


\section{MAIN RESULT}

Adding an input $u(t)$ to a switched homogenous DAE system, may change the behaviour of the system in such a way that the averaging result is not consistent; in the limit the averaged solution does not converge to the switched solution, as shown in the following numerical example.

Example 1: Consider an homogeneous switched system with two modes described by the following matrices

$E_{1}=\left[\begin{array}{ll}1 & 0 \\ 0 & 0\end{array}\right] A_{1}=\left[\begin{array}{cc}1 & -1 \\ 0 & 2\end{array}\right] E_{2}=\left[\begin{array}{ll}1 & 0 \\ 0 & 1\end{array}\right] A_{2}=\left[\begin{array}{cc}3 & 4 \\ -2 & 1\end{array}\right]$,

where the consistency, differential and impulsive projectors of the first mode are given by

$$
\Pi_{1}=\left[\begin{array}{ll}
1 & 0 \\
0 & 0
\end{array}\right], \quad \Pi_{1}^{\text {diff }}=\left[\begin{array}{cc}
1 & \frac{1}{2} \\
0 & 0
\end{array}\right], \quad \Pi_{1}^{\mathrm{imp}}=\left[\begin{array}{cc}
0 & 0 \\
0 & \frac{1}{2}
\end{array}\right],
$$

while the second mode is described by an ODE then $\Pi_{2}=$ $\Pi_{2}^{\text {diff }}$ are identity matrices while $\Pi_{2}^{\mathrm{imp}}$ is a zero matrix.

The consistency projectors commute, hence the averaging result holds. Consider now the non-homogeneous system in the form (1) with the same matrix pairs $\left(E_{i}, A_{i}\right)$ reported above and the input matrices $B_{1}=\left[\begin{array}{ll}1 & 2\end{array}\right]^{\top}, B_{2}=\left[\begin{array}{ll}1 & 0\end{array}\right]^{\top}$. In Fig. 2 the trajectories of the switched system are shown when $u(t)=5$. It it can be seen that, although the first state variable seems to converge, the second state variable clearly does not converge for an increasing switching frequency.

It is of interest to analyze under which particular assumptions an averaged model for non-homogeneous DAEs is still consistent; when one includes the presence of an exogenous input starting from an homogeneous DAEs for which the averaging result holds. In particular we consider exogenous inputs which are Lipschitz.

Definition 4 (Lipschitz function): A function $f(\xi): \mathbb{R} \rightarrow$ $\mathbb{R}^{q}$ is Lipschitz if there exists a positive constant $\mathrm{L}$ such that for all $\xi_{1}, \xi_{2}$ the following inequality

$$
\left\|f\left(\xi_{1}\right)-f\left(\xi_{2}\right)\right\| \leq L\left\|\xi_{1}-\xi_{2}\right\|
$$

holds.

Theorem 1: Consider the regular switched DAE (1) with periodic switching of period $p>0$ and with corresponding consistency, differential and impulse projectors. Assume that

(i) the consistency projectors commute, i.e. (7),

(ii) the input $u(t): \mathbb{R} \rightarrow \mathbb{R}^{m}$ is Lipschitz with constant $L$, (iii) $\Pi_{i}^{\mathrm{imp}} B_{i}=0, i=1,2, \ldots, \mathrm{q}$.

Consider the following averaged model

$$
\begin{aligned}
\dot{x}_{\mathrm{av}}(t) & =A_{\mathrm{av}} x_{\mathrm{av}}(t)+B_{\mathrm{av}} u(t) \\
x_{\mathrm{av}}\left(0^{-}\right) & =\Pi_{\cap} x_{0},
\end{aligned}
$$

where

$$
B_{\mathrm{av}}:=\Pi_{\cap}\left(B_{1}^{\text {diff }} d_{1}+B_{2}^{\text {diff }} d_{2}+\ldots+B_{\mathrm{q}}^{\text {diff }} d_{\mathrm{q}}\right)
$$

$A_{\text {av }}, \Pi_{\cap}$ given by (9), and $x_{0} \in \mathbb{R}^{n}$. Consider an arbitrary constant $\Delta>p$, then the following holds

$$
x_{\sigma, p}(t)-x_{\mathrm{av}}(t)=\mathrm{O}(p), \forall t \in[p, \Delta]
$$
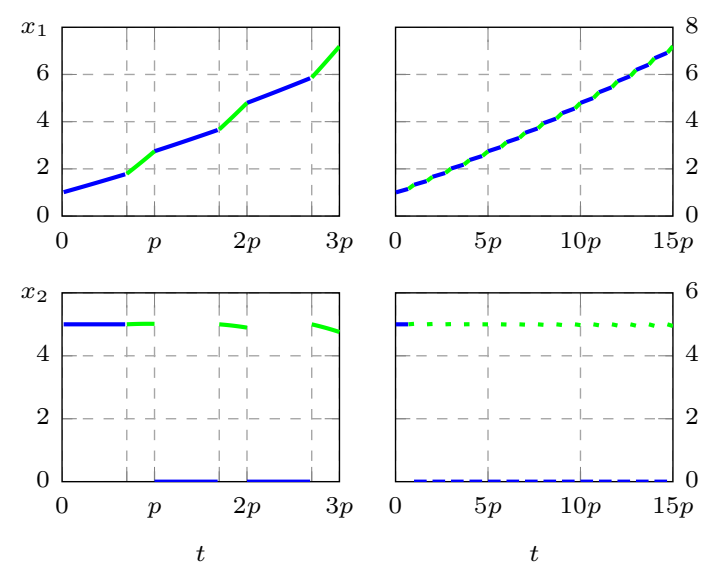

Fig. 2. Time evolutions of the state components for slow switching ( $p=$ $0.1 s$, left) and fast switching ( $p=0.02 s$, right). The trajectories of the switched DAE are colored according to the active mode (mode 1 blue, mode 2 green).

where $x_{\sigma, p}$ and $x_{a v}$ are the solutions of the switched and of the averaged systems, respectively.

In Section IV the feasibility of the assumptions of Theorem 1 are verified for a practical electrical circuit. In order to prove the main result the following two lemmas are needed.

Lemma 1: Consider the switched DAEs (1) with switching period $p>0$, the corresponding flow matrices and consistency, differential and impulsive projectors. Assume that

$$
\Pi_{i}^{\mathrm{imp}} B_{i}=0, \quad i=1,2, \ldots, \mathrm{q} .
$$

Then the (impulse-free) solution behavior of (1) is equivalent to the solution behavior of the following switched ODE with jumps

$$
\begin{aligned}
\dot{x}(t) & =A_{i}^{\mathrm{diff}} x(t)+B_{i}^{\mathrm{diff}} u(t), \quad t \in\left(s_{k, i}, s_{k, i+1}\right) \\
x\left(s_{k, i}^{+}\right) & =\Pi_{i} x\left(s_{k, i}^{-}\right) \\
x\left(0^{-}\right) & =\Pi_{\cap} x_{0}
\end{aligned}
$$

where $B_{i}^{\text {diff }}=\Pi_{i}^{\text {diff }} B_{i}$ and the switching times are given by (4).

Proof: The proof directly follows by considering the solution (5) of a non-homogeneous DAE together with (13).

Lemma 2: Consider the following functions

$$
\begin{aligned}
\mathcal{G}(p)\{u\} & =\sum_{i=1}^{\mathrm{q}} F_{\mathrm{q}} \cdots F_{i+1} Q_{i} u\left(\alpha_{i}\right) d_{i} p \\
\mathcal{G}_{\mathrm{av}}(p)\{u\} & =Q_{\mathrm{av}} u\left(\alpha_{q+1}\right) p
\end{aligned}
$$

with $F_{i} \in \mathbb{R}^{n \times n}, Q_{i}, Q_{\mathrm{av}} \in \mathbb{R}^{n \times m}, i=1, \ldots, \mathrm{q}, \quad \mathrm{q} \in \mathbb{N}$, $\alpha_{i} \in \mathbb{R}$.

Assume that

(i) $u(t): \mathbb{R} \rightarrow \mathbb{R}^{m}$ is Lipschitz with constant $L$,

(ii) $F_{i}$ commute, i.e. $F_{i} F_{j}=F_{j} F_{i}, i \neq j \in\{1, \ldots, q\}$,

(iii) $Q_{\mathrm{av}}=F_{\cap}\left(Q_{1} d_{1}+Q_{2} d_{2}+\ldots Q_{\mathrm{q}} d_{\mathrm{q}}\right), \quad F_{\cap}=F_{\mathrm{q}} \cdots F_{1}$,

(iv) $\left|\alpha_{i}-\alpha_{\mathrm{q}+1}\right| \leq \beta_{i} p$ with $\beta_{i}$ constants, $i=1, \ldots, q$.

Then

$$
F_{\cap} \mathcal{G}(p)\{u\}-\mathcal{G}_{\mathrm{av}}(p)\{u\}=\mathrm{O}\left(p^{2}\right) .
$$


Proof: Due to the commutativity property one has that

$$
\begin{aligned}
\left\|F_{\cap} \mathcal{G}(p)\{u\}-\mathcal{G}_{\mathrm{av}}(p)\{u\}\right\|= & \left\|\sum_{i=1}^{\mathrm{q}}\left(F_{\cap} Q_{i} u\left(\alpha_{i}\right) d_{i} p\right)-Q_{\mathrm{av}} u\left(\alpha_{\mathrm{q}+1}\right) p\right\| \\
= & \sum_{i=1}^{\mathrm{q}} \|\left(F_{\cap} Q_{i} u\left(\alpha_{i}\right) d_{i} p-F_{\cap} Q_{i} u\left(\alpha_{\mathrm{q}+1}\right) d_{i} p \|\right. \\
& =\sum_{i=1}^{\mathrm{q}}\left\|F_{\cap} Q_{i}\right\|\left\|u\left(\alpha_{i}\right)-u\left(\alpha_{\mathrm{q}+1}\right)\right\| d_{i} p \\
& \leq \sum_{i=1}^{\mathrm{q}}\left\|F_{\cap} Q_{i}\right\| L\left\|\alpha_{i}-\alpha_{\mathrm{q}+1}\right\| d_{i} p \\
& \leq \sum_{i=1}^{\mathrm{q}}\left\|F_{\cap} Q_{i}\right\| L d_{i} \beta_{i} p^{2}
\end{aligned}
$$

Proof of Theorem 1. Consider the solution of the switched ODE (14) on the interval $\left(s_{k-1, \mathrm{q}}, t_{k}\right)$ evaluated at $t_{k}^{-}$:

$$
x\left(t_{k}^{-}\right)=e^{A_{\mathrm{q}}^{\text {diff }} d_{\mathrm{q}} p} x\left(s_{k-1, \mathrm{q}}^{+}\right)+\int_{s_{k-1, \mathrm{q}}}^{t_{k}} e^{A_{\mathrm{q}}^{\text {diff }}\left(t_{k}-\xi\right)} B_{\mathrm{q}}^{\text {diff }} u(\xi) \mathrm{d} \xi
$$

where

$$
x\left(s_{k-1, \mathrm{q}}^{+}\right)=\Pi_{\mathrm{q}} x\left(s_{k-1, \mathrm{q}}^{-}\right)
$$

and $x\left(s_{k-1, q}^{-}\right)$is the solution on the interval $\left(s_{k-1, \mathrm{q}-1}, s_{k-1, \mathrm{q}}\right) \quad$ evaluated at $s_{k-1, \mathrm{q}}^{-}$. Substituting the solution $x\left(s_{k-1, q}^{-}\right)$in (15) and in (16) and iterating for all $q$ modes one obtains a linear discrete time system for the interval $\left(t_{k-1}, t_{k}\right)$

$$
x\left(t_{k}^{-}\right)=H(p) x\left(t_{k-1}^{-}\right)+\mathcal{I}(p)\left\{u_{k-1}\right\}
$$

where $u_{k-1}$ is the input in the time interval $\left(t_{k-1}, t_{k}\right)$ translated into the time interval $(0, p)$, i.e., $u_{k-1}:[0, p] \rightarrow$ $\mathbb{R}^{m}$ with $\xi \mapsto u\left(\xi+t_{k-1}\right)$, and

$$
\begin{aligned}
H(p) & =\prod_{i=1}^{q} e^{A_{i}^{\mathrm{diff}} d_{i} p} \Pi_{i} \\
\mathcal{I}(p)\left\{u_{k-1}\right\} & =\sum_{i=1}^{q} \prod_{j=i+1}^{q} e^{A_{j}^{\mathrm{diff}} d_{j} p} \Pi_{j} \int_{c_{i-1}}^{c_{i}} e^{A_{i}^{\mathrm{diff}}\left(c_{i}-\xi\right)} B_{i}^{\mathrm{diff}} u_{k-1}(\xi) \mathrm{d} \xi
\end{aligned}
$$

where the following notation has been adopted

$$
\prod_{j=k}^{\ell} M_{j}:=M_{\ell} M_{\ell-1} \cdots M_{k+1} M_{k}, \quad c_{i}=\sum_{j=1}^{i} d_{j} p, c_{0}=0
$$

with $i=1,2, \ldots, q$. The solution of (17) for all $k \in \mathbb{N}$ is

$$
x\left(t_{k}^{-}\right)=H(p)^{k} x_{0}+\sum_{i=0}^{k-1} H(p)^{k-1-i} \mathcal{I}(p)\left\{u_{i}\right\} .
$$

Consider the solution of the averaged system (11) in the interval $\left(t_{k-1}, t_{k}\right)$ then for all $k \in \mathbb{N}$ one as

$$
x_{\mathrm{av}}\left(t_{k}^{-}\right)=H_{\mathrm{av}}(p)^{k} \Pi_{\cap} x_{0}+\sum_{i=0}^{k-1} H_{\mathrm{av}}(p)^{k-1-i} \mathcal{I}_{\mathrm{av}}(p)\left\{u_{i}\right\}
$$

with

$$
H_{\mathrm{av}}(p)=e^{A_{\mathrm{av}} p}, \quad \mathcal{I}_{\mathrm{av}}(p)\left\{u_{k-1}\right\}=\int_{0}^{p} e^{A_{\mathrm{av}}(p-\xi)} B_{\mathrm{av}} u_{k-1}(\xi) \mathrm{d} \xi .
$$

Considering the Taylor approximation of the exponential matrix [7], one obtains

$$
\begin{aligned}
H(p)= & \Pi_{\cap}+\mathrm{O}(p), \quad H_{\mathrm{av}}(p)=I+\mathrm{O}(p) \\
\mathcal{I}(p)\left\{u_{k-1}\right\}= & \sum_{i=1}^{\mathrm{q}}\left[\left(\Pi_{\mathrm{q}} \Pi_{\mathrm{q}-1} \cdots \Pi_{i+1}+\mathrm{O}(p)\right) \int_{c_{i-1}}^{c_{i}}(I\right. \\
& \left.+\mathrm{O}(p)) B_{i}^{\text {diff }} u_{k-1}(\xi) \mathrm{d} \xi\right], \\
\mathcal{I}_{\text {av }}(p)\left\{u_{k-1}\right\}= & \int_{0}^{p}(I+\mathrm{O}(p-\xi)) B_{\mathrm{av}} u_{k-1}(\xi) d \xi
\end{aligned}
$$

where we used that $\mathrm{O}\left(c_{i}-\xi\right)$ can be substituted by $\mathrm{O}(p)$ since $\left(c_{i}-\xi\right) \leq p$, for all $i=1,2, \ldots, \mathrm{q}$.

Furthermore taking into account that $\frac{1}{b-a} \int_{a}^{b} f(t) \mathrm{d} t=$ $f(\alpha)$, with $\alpha \in[a, b]$ one has

$$
\mathcal{I}(p)\left\{u_{k-1}\right\}=\sum_{i=1}^{\mathrm{q}} \Pi_{\mathrm{q}} \cdots \Pi_{i+1} B_{i}^{\text {diff }} u_{k-1}\left(\alpha_{i}\right) d_{i} p+\mathrm{O}\left(p^{2}\right)
$$

$\mathcal{I}_{\mathrm{av}}(p)\left\{u_{k-1}\right\}=B_{\mathrm{av}} u_{k-1}\left(\alpha_{p+1}\right) p+\mathrm{O}\left(p^{2}\right)$

where $\alpha_{i} \in\left[c_{i-1}, c_{i}\right]$ and $\alpha_{\mathrm{q}+1} \in[0, p]$.

Invoking [26, Lemma 2] one has that $H(p)^{i}$ and $H_{\mathrm{av}}(p)^{i}$ are $\mathrm{O}(1)$ functions for each $i \in \mathfrak{I}$. Further noting that $\mathcal{I}(p)\left\{u_{i}\right\}$ and $\mathcal{I}_{a v}(p)\left\{u_{i}\right\}$ are $\mathrm{O}(p)$ functions and taking into account the averaging result (10) and $k=\mathrm{O}(1 / p)$ one obtains

$$
\begin{aligned}
\left\|x\left(t_{k}\right)-x_{\mathrm{av}}\left(t_{k}\right)\right\| \leq & \beta_{1} p+\sum_{i=0}^{k-1} \| H(p)^{k-2-i} \Pi_{\cap} \mathcal{I}(p)\left\{u_{i}\right\} \\
& -H_{\mathrm{av}}(p)^{k-2-i} \mathcal{I}_{\mathrm{av}}(q)\left\{u_{i}\right\} \| .
\end{aligned}
$$

where $\beta_{1}$ is a constant. For each $i$ one can write

$$
\begin{aligned}
\| H(p)^{i} \Pi_{\cap} & \mathcal{I}(p)\left\{u_{i}\right\}-H_{\mathrm{av}}(p)^{i} \mathcal{I}_{\mathrm{av}}(q)\left\{u_{i}\right\} \|= \\
& \| H(p)^{i} \Pi_{\cap} \mathcal{I}(p)\left\{u_{i}\right\}-H_{\mathrm{av}}(p)^{i} \Pi_{\cap} \mathcal{I}(p)\left\{u_{i}\right\} \\
& +H_{\mathrm{av}}(p)^{i} \Pi_{\cap} \mathcal{I}(p)\left\{u_{i}\right\}-H_{\mathrm{av}}(p)^{i} \mathcal{I}_{\mathrm{av}}(q)\left\{u_{i}\right\} \| \\
\leq & \left\|\left(H(p)^{i}-H_{\mathrm{av}}(p)^{i} \Pi_{\cap}\right) \Pi_{\cap} \mathcal{I}(p)\left\{u_{i}\right\}\right\| \\
& +\left\|H_{\mathrm{av}}(p)^{i}\left(\Pi_{\cap} \mathcal{I}(p)\left\{u_{i}\right\}-\mathcal{I}_{\mathrm{av}}(p)\left\{u_{i}\right\}\right)\right\| .
\end{aligned}
$$

Due to the averaging result (10) and invoking Lemma 2 and [26, Lemma 2] one obtains that

$$
H(p)^{i} \Pi_{\cap} \mathcal{I}(p)\left\{u_{i}\right\}-H_{\mathrm{av}}(p)^{i} \mathcal{I}_{\mathrm{av}}(q)\left\{u_{i}\right\}=\mathrm{O}\left(p^{2}\right) .
$$

Combining (23) and (24) the proof holds.

The Lipschitz assumption on the input $u(t)$ can be relaxed in the case of a switched non-homogeneous DAE with two modes so as shown by the following result.

Proposition 3: Consider a switched DAE with two modes where the assumption Theorem 1-(iii) and the averaging 
result for the relative homogeneous system (10) hold. Then if the following conditions holds

$$
B_{2}^{\text {diff }}=B_{1}^{\text {diff }},
$$

the averaged condition (12) is satisfied.

Proof: The solution of the switched ODE system (14) and of the averaged system (11) can be written as (19) and (20) respectively, then considering the averaged result (10) and [26, Lemma 2] one has:

$\left\|x\left(t_{k}\right)-x_{\text {av }}\left(t_{k}\right)\right\| \leq \beta_{1} p+\sum_{i=0}^{k-1}\left\|\Pi_{\cap} \mathcal{I}(p)\left\{u_{i}\right\}-\mathcal{I}_{\text {av }}(p)\left\{u_{i}\right\}\right\|$,

where $\beta_{1}$ is a constant and $\mathcal{I}(p)\left\{u_{i}\right\}$ and $\mathcal{I}_{\text {av }}(p)\left\{u_{i}\right\}$ are given by (22). Indeed, taking into account $k=\mathrm{O}(1 / p)$

$$
\begin{aligned}
& \left\|x\left(t_{k}\right)-x_{\mathrm{av}}\left(t_{k}\right)\right\| \leq \sum_{i=0}^{k-1} \| \Pi_{\cap} \Pi_{2} \int_{0}^{d_{1} p} B_{1}^{\text {diff }} u_{i}(\xi) \mathrm{d} \xi \\
& \quad+\int_{d_{1} p}^{p} \Pi_{\cap} B_{2}^{\text {diff }} u_{i}(\xi) \mathrm{d} \xi \\
& \quad-\int_{0}^{p} B_{\text {av }} u_{i}(\xi) \mathrm{d} \xi \|+\beta_{2} p \\
& =\sum_{i=0}^{k-1} \| \int_{d_{1} p}^{p}\left(\Pi_{\cap} B_{2}^{\text {diff }}-\Pi_{\cap} B_{2}^{\text {diff }} d_{2}\right. \\
& \left.\quad-\Pi_{\cap} B_{1}^{\text {diff }} d_{1}\right) u_{i}(\xi) \mathrm{d} \xi+\int_{0}^{d_{1} p}\left(\Pi_{\cap} \Pi_{2} B_{1}^{\text {diff }}\right. \\
& \left.\quad-\Pi_{\cap} \Pi_{2} B_{1}^{\text {diff }} d_{1}-\Pi_{\cap} B_{2}^{\text {diff }} d_{2}\right) u_{i}(\xi) \mathrm{d} \xi \|+\beta_{2} p \\
& =\sum_{i=0}^{k-1} \| \Pi_{\cap}\left(B_{2}^{\text {diff }}-B_{1}^{\text {diff }}\right) d_{1} \int_{d_{1} p}^{p} u_{i}(\xi) \mathrm{d} \xi \\
& \quad+\Pi_{\cap}\left(B_{2}^{\text {diff }}-B_{1}^{\text {diff }}\right) d_{2} \int_{0}^{d_{1} p} u_{i}(\xi) \mathrm{d} \xi \|+\beta_{2} p
\end{aligned}
$$

then if $B_{2}^{\text {diff }}=B_{1}^{\text {diff }}$ the proof holds.

\section{ILLUSTRATIVE EXAMPLE}

Consider the switched capacitor circuit shown in Figure 3. By applying the Kirchhoff's laws to the four different configurations obtained by combining the different states of the two ideal switches, the system can be described as a switched DAEs where the state variables are the voltage on the two capacitors and the current through the inductor respectively, $x=\left[v_{C_{1}}, v_{C_{2}}, i_{L}\right]^{\top}$. Note that the system is non-homogeneous and the assumptions of Theorem 1 hold.

The matrices $\left(E_{i}, A_{i}, B_{i}\right)$ are given by

$$
\begin{aligned}
E_{1}=\left[\begin{array}{ccc}
C_{1} & 0 & 0 \\
0 & C_{2} & 0 \\
0 & 0 & L
\end{array}\right] & A_{1}=\left[\begin{array}{ccc}
0 & 0 & 1 \\
0 & -\frac{1}{R_{2}} & 0 \\
1 & 0 & -R_{1}
\end{array}\right] & B_{1}=\left[\begin{array}{l}
0 \\
0 \\
1
\end{array}\right], \\
E_{2}=\left[\begin{array}{ccc}
C_{1} & C_{2} & 0 \\
0 & 0 & L \\
0 & 0 & 0
\end{array}\right] & A_{2}=\left[\begin{array}{ccc}
0 & -\frac{1}{R_{2}} & 1 \\
-1 & 0 & -R_{1} \\
1 & -1 & 0
\end{array}\right] & B_{2}=\left[\begin{array}{l}
0 \\
1 \\
0
\end{array}\right], \\
E_{3}=\left[\begin{array}{ccc}
C_{1} & C_{2} & 0 \\
0 & 0 & 0 \\
0 & 0 & 0
\end{array}\right] & A_{3}=\left[\begin{array}{ccc}
0 & -\frac{1}{R_{2}} & 0 \\
1 & -1 & 0 \\
0 & 0 & 1
\end{array}\right] & B_{3}=\left[\begin{array}{l}
0 \\
0 \\
0
\end{array}\right], \\
E_{4}=\left[\begin{array}{ccc}
C_{1} & 0 & 0 \\
0 & C_{2} & 0 \\
0 & 0 & 0
\end{array}\right] & A_{4}=\left[\begin{array}{ccc}
0 & 0 & 0 \\
0 & -\frac{1}{R_{2}} & 0 \\
0 & 0 & 1
\end{array}\right] & B_{4}=\left[\begin{array}{l}
0 \\
0 \\
0
\end{array}\right] .
\end{aligned}
$$

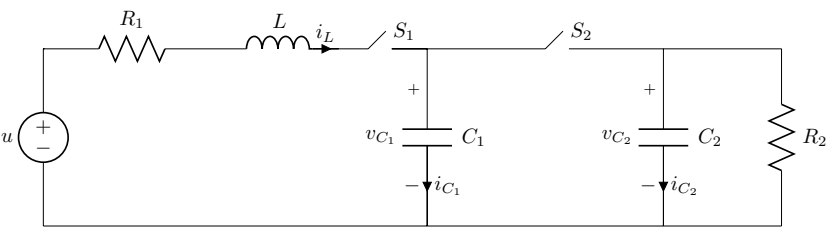

Fig. 3. Electrical circuit with two capacitors and one inductor.

where the $i$-th mode is defined according to the positions of the switches $S_{1}, S_{2}$. In particular, the modes $i=$ $1,2,3,4$ correspond to the switches pairs $\left\{S_{1}, S_{2}\right\}$ in the states $\{$ closed, open $\},\{$ closed, closed $\},\{$ open, closed $\}$ and $\{$ open, open $\}$, respectively.

Define the constants $\rho_{1}=\frac{C_{1}}{C_{1}+C_{2}}$ and $\rho_{2}=\frac{C_{2}}{C_{1}+C_{2}}$. The consistency projectors are

$$
\begin{aligned}
& \Pi_{1}=\left[\begin{array}{lll}
1 & 0 & 0 \\
0 & 1 & 0 \\
0 & 0 & 1
\end{array}\right], \\
& \Pi_{3}=\left[\begin{array}{ccc}
\rho_{1} & \rho_{2} & 0 \\
\rho_{1} & \rho_{2} & 0 \\
0 & 0 & 0
\end{array}\right], \\
& \Pi_{2}=\left[\begin{array}{ccc}
\rho_{1} & \rho_{2} & 0 \\
\rho_{1} & \rho_{2} & 0 \\
0 & 0 & 1
\end{array}\right], \\
& \Pi_{4}=\left[\begin{array}{lll}
1 & 0 & 0 \\
0 & 1 & 0 \\
0 & 0 & 0
\end{array}\right] .
\end{aligned}
$$

The consistency projectors commute then the averaged model for the homogeneous part is consistent. The conditions Theorem 1-(iii) hold where

$$
\begin{array}{rlrl}
\Pi_{1}^{\text {imp }} & =\left[\begin{array}{ccc}
0 & 0 & 0 \\
0 & 0 & 0 \\
0 & 0 & 0
\end{array}\right], & \Pi_{2}^{\mathrm{imp}}=\left[\begin{array}{ccc}
0 & 0 & \rho_{2} \\
0 & 0 & -\rho_{1} \\
0 & 0 & 0
\end{array}\right], \\
\Pi_{3}^{\mathrm{imp}}=\left[\begin{array}{ccc}
0 & \rho_{2} & 0 \\
0 & -\rho_{1} & 0 \\
0 & 0 & 1
\end{array}\right], & \Pi_{4}^{\mathrm{imp}}=\left[\begin{array}{lll}
0 & 0 & 0 \\
0 & 0 & 0 \\
0 & 0 & 1
\end{array}\right] .
\end{array}
$$

The matrices of the averaged system are

$$
A_{\mathrm{av}}=\left[\begin{array}{ccc}
-\frac{\rho_{1}^{2}}{R_{2} C_{1}}-\frac{\rho_{2}^{2}}{R_{2} C_{1}} & 0 \\
-\frac{\rho_{1}^{2}}{R_{2} C_{1}}-\frac{\rho_{2}^{2}}{R_{2} C_{1}} & 0 \\
0 & 0 & 0
\end{array}\right], \quad B_{\mathrm{av}}=0, \Pi_{\cap}=\left[\begin{array}{ccc}
\rho_{1} & \rho_{2} & 0 \\
\rho_{1} & \rho_{2} & 0 \\
0 & 0 & 0
\end{array}\right] .
$$

Note that the averaged model is independent from the input due to the particular structure of $\Pi_{\cap}$.

For the simulations the following parameters have been used: $C_{1}=80.36 \mathrm{mF}, C_{2}=8.2 \mathrm{mF}, L=5 \mathrm{H}, R_{2}=$ $20 \Omega, \quad R_{1}=10 \Omega$ and $\bar{u}=5 \mathrm{~V}$ with duty cycles $\left(d_{1}, d_{2}, d_{3}, d_{4}\right)=(0.3,0.4,0.2,0.1)$ and initial conditions $x_{0}=(1,1,0)^{\top}$. The evolutions of the state variables in the same time interval equal to $0.3 \mathrm{~s}$ and for two different switching periods are reported in Fig. 4. By comparing the left and right sides of the figure it is evident that by reducing the period, the error between the switched and the averaged solutions decreases for all state components.

\section{CONCLUSIONS}

The averaging problem for switched systems with commutations between multi-modes, each one described by nonhomogeneous linear differential algebraic equations (DAEs) has been considered. It is shown that an averaged approach is possible and the solution of the switched system converges to the averaged system with an error of the order of the switching period. A switched electrical circuit motivates the practical interest of the proposed analysis. A possible direction for future research is the extension of the averaging result to the case of presence of jumps in the input. 

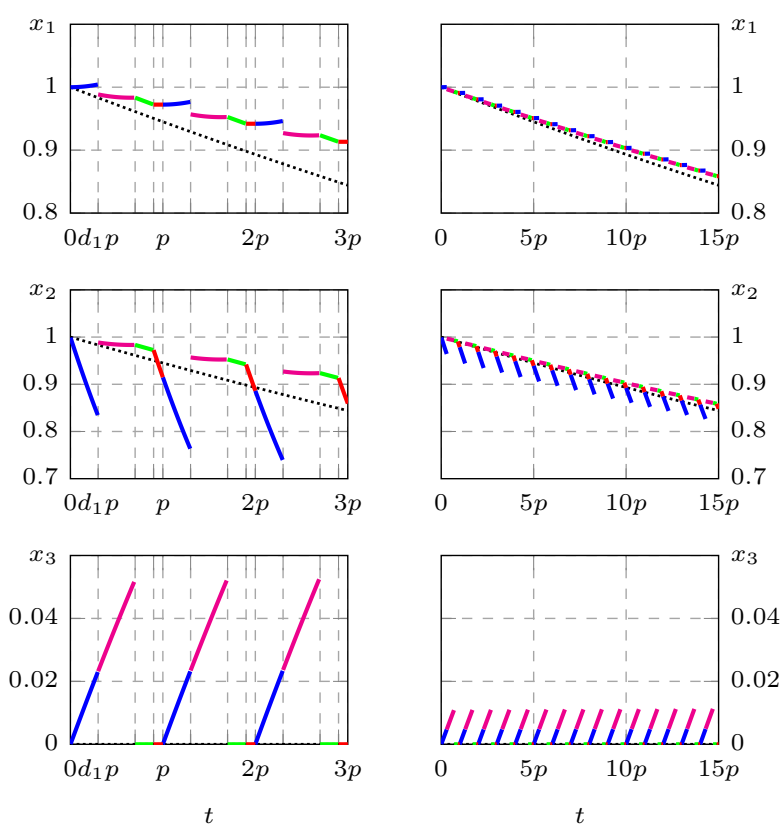

Fig. 4. Evolution of the state variables (first component top, second component middle, third component bottom)for slow switching ( $p=0.1 s$, left) and fast switching ( $p=0.02 s$, right). The averaging dynamics are plotted with dotted black lines, while the trajectories of the switched DAE are colored according to the active mode (mode 1 blue, mode 2 magenta, mode 3 green, mode 4 red).

\section{REFERENCES}

[1] S. Moberg and S. Hanssen, "A DAE approach to feedforward control of flexible manipulators," in IEEE International Conference on Robotics and Automation, Rome, Italy, Apr. 2007, pp. 3439-3444.

[2] N. Villa-Villasenor, G. Gonzalez-Avalos, and J. Rico-Melgoza, "Bond graph modeling, DAE formulation and numeric simulation of a three phase rectifier," in 17th International Conference on Methods and Models in Automation and Robotics, Miedzyzdrojie, Poland, Aug. 2012, pp. 458-463.

[3] W. Marszalek and Z. W. Trzaska, "DAE models of electrical power systems and their bifurcations around singularities," in 43rd IEEE Conference on Decision and Control, Dec. 2004, pp. 4833-4838.

[4] J. Zhang, G. Zhang, and L. Zhu, "Feedback control for singularity induced bifurcation of a differential-algebraic biological economic system," in International Conference on Information Science and Technology, Hubei, China, Mar. 2012, pp. 1-4.

[5] F. Vasca and L. Iannelli, (Eds.), Dynamics and Control of Switched Electronic Systems. London,UK: Springer, 2012.

[6] A. D. Domínguez-García and S. Trenn, "Detection of impulsive effects in switched DAEs with application to power electronics reliability analysis," in 49th IEEE Conference on Decision and Control, Atlanta, GA, USA, Dec. 2010, pp. 5662-5667.

[7] J. Ezzine and A. H. Haddad, "Error bounds in the averaging of hybrid systems," IEEE Transactions on Automatic Control, vol. 34, no. 11, pp. 1188-1192, 1989.

[8] L. Iannelli, K. Johansson, U. Jönsson, and F. Vasca, "Averaging of nonsmooth systems using dither," Automatica, vol. 42, no. 4, pp. 669676, 2006.

[9] M. Porfiri, D. G. Roberson, and D. J. Stilwell, "Tracking and formation control of multiple autonomous agents: a two level consensus approach," Automatica, vol. 43, no. 8, pp. 1318-1328, 2007.

[10] D. J. Stilwell, E. M. Bollt, and D. G. Roberson, "Sufficient conditions for fast switching synchronization in time-varying network topologies," SIAM Journal on Applied Dynamical Systems, vol. 5, no. 1, pp. 140-156, 2006.

[11] M. Porfiri and F. Fiorilli, "Global pulse synchronization of chaotic oscillators through fast-switching: theory and experiments," Chaos, Solitons and Fractals, vol. 41, no. 1, pp. 245-262, 2009.
[12] X. Shen, J. Zhang, E. J. Barth, and M. Goldfarb, "Nonlinear averaging applied to the control of pulse-width modulated (PWM) pneumatic systems," in American Control Conference, Boston, Massachussetts, USA, 2004, pp. 4444-4448.

[13] A. K. Gelig and A. N. Churilov, Stability and Oscillations of Nonlinear Pulse-modulated Systems. Boston, Massachussetts, USA: Birkhäuser, 1998.

[14] B. Sedghi, B. Srinivasav, and R. Longchamp, "Control of hybrid systems via dehybridization," in American Control Conference, Anchorage, Alaska, USA, 2002, pp. 692-697.

[15] R. Marquez, E. Altman, and S. Solé-Álvarez, "Time-averaging of high-speed data transfer protocol," IEEE Transactions on Automatic Control, vol. 50, no. 12, pp. 2065-2069, 2005.

[16] H. Sira-Ramírez, M. Zribi, and S. Ahmad, "Pulse width modulated control of robotic manipulators," in American Control Conference, Honolulu, Hawaii, 1990, pp. 336-367.

[17] M. Porfiri and F. Fiorilli, "Experiments on node-to-node pinning control of Chua's circuits," Physica D: Nonlinear Phenomena, vol. 239, no. 8, pp. 454-464, 2010.

[18] I. Belykh, M. di Bernardo, J. Kurths, and M. Porfiri, "Evolving dynamical networks," Physica D Nonlinear Phenomena, vol. 267, pp. $1-6,2014$.

[19] A. Codrean and T. L. Dragomir, "Averaged modeling of the cardiovascular system," in 52nd IEEE Annual Conference on Decision and Control, Florence, Italy, Dec. 2013, pp. 2066-2071.

[20] S. Almér and U. Jönsson, "Harmonic analysis of pulse-width modulated systems," Automatica, vol. 45, no. 4, pp. 851-862, 2009.

[21] M. Porfiri, D. G. Roberson, and D. J. Stilwell, "Fast switching analysis of linear switched systems using exponential splitting," SIAM Journal of Control Optimization, vol. 47, no. 5, pp. 2582-2597, 2008.

[22] L. Iannelli, K. Johansson, U. Jönsson, and F. Vasca, "Subtleties in the averaging of a class of hybrid systems with applications to power converters," Control Engineering Practice, vol. 18, no. 8, pp. 961-975, 2008.

[23] A. Teel and D. Nešić, "Averaging theory for a class of hybrid systems," Dynamics of Continuous, Discrete and Impulsive Systems, vol. 17, no. 6, pp. 829-851, 2010.

[24] C. Pedicini, F. Vasca, L. Iannelli, and U. Jönsson, "An overview on averaging for pulse-modulated switched systems," in 50th IEEE Conference on Decision and Control and European Control Conference, Orlando, FL, USA, Dec. 2011, pp. 1860-1865.

[25] L. Iannelli, C. Pedicini, S. Trenn, and F. Vasca, "On averaging for switched linear differential algebraic equations," in 12th IEEE European Control Conference, Zurich, Switzerland, 2013, pp. 2163 -2168 .

[26] - "An averaging result for switched DAEs with multiple modes," in 52nd IEEE Conference on Decision and Control Conference, Florence, Italy, Dec. 2013, pp. 1378-1383.

[27] E. Mostacciuolo, S. Trenn, and F. Vasca, "Partial averaging for switched DAEs with two modes," in 14th IEEE annual European Control Conference, Linz, Austria, Jul. 2015, to be presented.

[28] K. Weierstraß, Zur Theorie der linearen und quadratischen Formen. Berlin: Monatsberichte der Koniglich Preuischen Akademie der Wissenschaften zu Berlin, 1868.

[29] T. Berger, A. Ilchmann, and S. Trenn, "The quasi-Weierstraß form for regular matrix pencils," Linear Algebra and its Applications, vol. 436, no. 10, pp. 4052-4069, 2012.

[30] V. A. Armentano, "The pencil (sE-A) and controllabilityobservability for generalized linear systems: a geometric approach," SIAM Journal on Control Optimization, vol. 24, pp. 616-638, 1986.

[31] K. Wong, "The eigenvalue problem $\lambda T x+S x$." Journal of differential Equations, vol. 16, pp. 270-280, 1974.

[32] A. Tanwani and S. Trenn, "On observability of switched differentialalgebraic equations," in 49th IEEE Conference on Decision and Control, Atlanta, GA, USA, Dec. 2010, pp. 5656-5661.

[33] S. Trenn, Distributional differential algebraic equations. Ilmenau: Univ.-Verlag, 2009

[34] - "Distributional averaging of switched DAEs with two modes," in 54th IEEE Annual Conference on Decision and Control, Osaka, Japan, Dec. 2015, to be presented. 